\title{
Signatures of red-shifted foot points in the quiescent coronal loop system
}

\author{
Yamini K. Rao ${ }^{1}$, Abhishek K. Srivastava ${ }^{1}$, Pradeep Kayshap ${ }^{2}$, and Bhola N. Dwivedi ${ }^{1}$ \\ ${ }^{1}$ Department of Physics, Indian Institute of Technology (BHU), Varanasi 221005, India \\ ${ }^{2}$ Institute of Physics, University of South Bohemia, Branišovská 1760, 37005 České Budějovice, Czech Republic
}

Correspondence: Abhishek K. Srivastava (asrivastava.app@itbhu.ac.in)

Received: 30 April 2019 - Discussion started: 9 May 2019

Accepted: 6 August 2019 - Published: 30 August 2019

\begin{abstract}
We observed quiescent coronal loops using multiwavelength observations from the Atmospheric Imaging Assembly (AIA) aboard the Solar Dynamics Observatory (SDO) on 13 April 2016. The flows at the foot points of such loop systems are studied using spectral data from the Interface Region Imaging Spectrograph (IRIS). The Doppler velocity distributions at the foot points lying in the moss region show the negligible or small flows at the $\mathrm{Ni}$ I, Mg II k3, and C II line corresponding to upper photospheric and chromospheric emissions. Significant red shifts (downflows) ranging from 1 to $7 \mathrm{kms}^{-1}$ are observed at $\operatorname{SiIV~(1393.78~} \AA^{\text {; }}$ $\log (T / K)=4.8)$, which is found to be consistent with the existing results regarding dynamical loop systems and moss regions. Such downflows agree well with the impulsive heating mechanism reported earlier.
\end{abstract}

\section{Introduction}

The active regions dominated by various loop structures are of significant importance for the study of coronal heating, since these loop systems act as a fundamental unit of the solar corona (Klimchuk, 2006; Reale, 2014; De Moortel and Browning, 2015). Moss is generally associated with plage regions around the active regions (Fletcher and De Pontieu, 1999), and transition region (TR) emission of hot core loops will provide us with a better understanding of the flows and thus the energy-transfer mechanism between the TR and corona.

Klimchuk (2006) provided a full review of the coronal heating problem. It describes how the coronal heating mechanisms are impulsive when explored from the perspective of elemental magnetic flux strands. It has also been well established that the loop structures emit significantly in the solar corona, which has been classified depending on their temperatures. The spectral studies of these loop systems in response to the Doppler shift provide a clue for distinguishing between the steady and impulsive heating mechanism (Del Zanna, 2008; Brooks et al., 2011).

Various types of loops are hot core loops (Del Zanna, 2008), warm loops (Del Zanna et al., 2011), fan loops (Young et al., 2012; Warren and Brooks, 2009, and references therein), and cool loops (Huang et al., 2015; Rao et al., 2019) present in the different regions of the solar atmosphere. The temperature and density diagnostics of quiescent coronal loops have been studied earlier to some extent (Del Zanna and Mason, 2003). However, there have not been many observations regarding the flows in the resolved strands or flux tubes of such loops in the solar corona.

In this paper, we study quiescent coronal loops with big loop arches that have one of their foot points anchored at the edges of moss region. The different strands in such large loop systems were identified using high-resolution observations of SDO-AIA (Solar Dynamics Observatory - Atmospheric Imaging Assembly), and the flows in it were studied, mapping the foot points to the lower region of the solar atmosphere. Section 2 describes the observational data and their analyses, presenting the details of the data used for our analyses. In Sect. 3, the results have been reported with their interpretations. In the last section, the discussions and conclusions are summarized. 


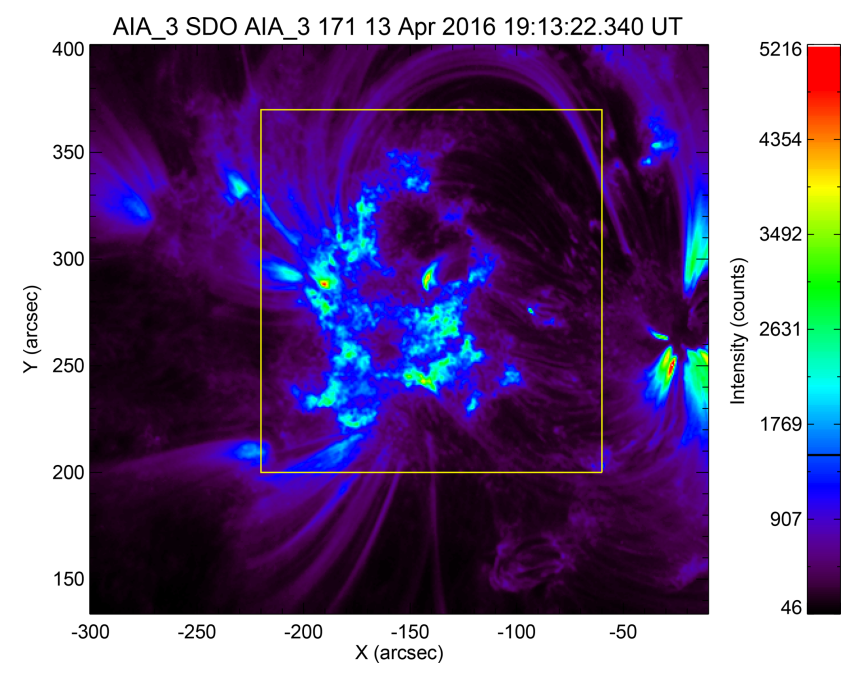

Figure 1. Intensity emission due to $171 \AA$ wavelength of SDO-AIA at 19:13:22 UT. The yellow box is overlaid to show the region of interest (ROI) taken to analyze the flows at the foot points of quiescent coronal loops.

\section{Observational data}

The Interface Region Imaging Spectrograph (IRIS) provides spectral data in the two UV domains: the FUV band (1331.7 to $1358.4 \AA$ and 1389.0 to $1407.0 \AA)$ and NUV band $(2782.7$ to $2835.1 \AA$ ), with a large number of spectral lines covering the photosphere, chromosphere, TR, and inner corona. Level 2 data are used for our study which are calibrated for the dark current removal and flat fielding effect (De Pontieu et al., 2014). We utilized Si IV (1393.78 $)$ ) Mg II k (2796.20 ̊), C II (1334.53 $)$ ), and Ni I (2799.47 ̊) spectral lines.

In our present work, we used dense raster data from IRIS for the time period 19:19:09 to 20:21:14 UTC on 13 April 2016, targeting the evolution of AR 12529 with a slit width of 0.35 in and a step cadence of $9.3 \mathrm{~s}$, covering the field of view of $141^{\prime \prime}$ in the $x$ direction and 175 in in the $y$ direction and centered at the coordinates $\left(X_{\text {cen }}, Y_{\text {cen }}\right)=4$ (-173 in, $275 \mathrm{in})$. The data are compensated for oscillations due to thermal variation using iris_orbitvarr_corr_12.pro in the SSWIDL library. The rest wavelengths for different spectral lines used in our analysis are calibrated using neutral lines from the relatively quiet-Sun area of the raster. The rest wavelength of Ni I used is $2944.4697 \AA$ A Mg II k was calibrated with respect to NiI, which is found to be $2796.3574 \AA$. The Si IV line is calibrated with respect to the Fe I (1392.8052 ̊) line, and C II is calibrated with respect to O I (1355.5987 $\AA$ ). Thus, the calibrated wavelengths used for our analysis are 1393.7604 and $1334.5406 \AA$ for Si IV and C II respectively.

The Doppler velocities are deduced using different spectral lines, i.e., Ni I 2799.47 ̊, Mg II k 2796.20 ̊, C II $1334.53 \AA$, and Si IV $1393.78 \AA$, respectively associated with the formation temperature of $\log (T / K)=4.2,4.0,4.3$, and 4.8. The velocity resolution of IRIS is $1 \mathrm{~km} \mathrm{~s}^{-1}$ (De Pontieu et al., 2014).

Si IV shows the characteristics of an optically thin line and is thus fitted with the single Gaussian curve, while NiI is the absorption line and the inverse Gaussian curve is fitted. Mg II k and C II are fitted with single or double Gaussian, depending on their profile characteristics.

The corresponding SDO-AIA observations are also taken in the different filters covering the UV-EUV range, corresponding to a different temperature range in the solar atmosphere. AIA provides full-disk multi-wavelength observations of coronal lines, with a spatial resolution of $1.5 \mathrm{in}$, a pixel size of $0.6 \mathrm{in}$, and a temporal cadence of $12 \mathrm{~s}$ (Lemen et al., 2012).

The co-aligned Level 2 SDO-AIA data cube was used in which all the wavelengths are matched to the field of view of $1600 \AA$. To co-align with the raster images of Si IV (1393.78 $)$, the near-time $171 \AA$ Amage properly crosscorrelated with $1600 \AA$ was used to compensate for the different resolution of two instruments. However, in our paper, we study the bulk plasma flows from the chosen moss region (in various boxes) by deducing the integrated spectral line profiles of various IRIS lines. The plage regions are identified in SDO-AIA image data, and the location is mapped onto comparatively high-resolution IRIS data.

\section{Observational results}

Figure 1 shows the intensity emission of AR 12529, where the plage region and various loops anchored in the moss region are visible in the $171 \AA$ wavelength of SDO-AIA. The green emission predominantly indicates the highest emission representing the part of the moss region. The yellow box is overlaid to show the region of interest (ROI).

In Fig. 2, the moss region has been identified with the brightest emission in the SDO-AIA $193 \AA$ filter. The intensity threshold of above 3000 counts (see Fig. 1), with values double those of the plage region surrounding it, was set, which is shown by contours overlaid over the different filters, corresponding to different temperature ranges from the upper photosphere to the corona. The northern segment of the moss regions where the quiescent coronal loops are anchored was further taken to analyze the flows at the foot points of these loop systems. The different bands of AIA show different morphological characteristics of an AR; 1600 and $1700 \AA$ represent the continuum emission at the upper photosphere, showing the plage region near the active region, and $304 \AA$ yields the chromospheric emission of the plage region. The hot loop structures are not distinctly visible. The middle row shows the inner coronal channels where quiescent coronal loops are clearly visible, and the moss region is identified. The last row shows the hot channels of SDOAIA $(335,211$, and $94 \AA$ ) where the quiescent loops taken for 


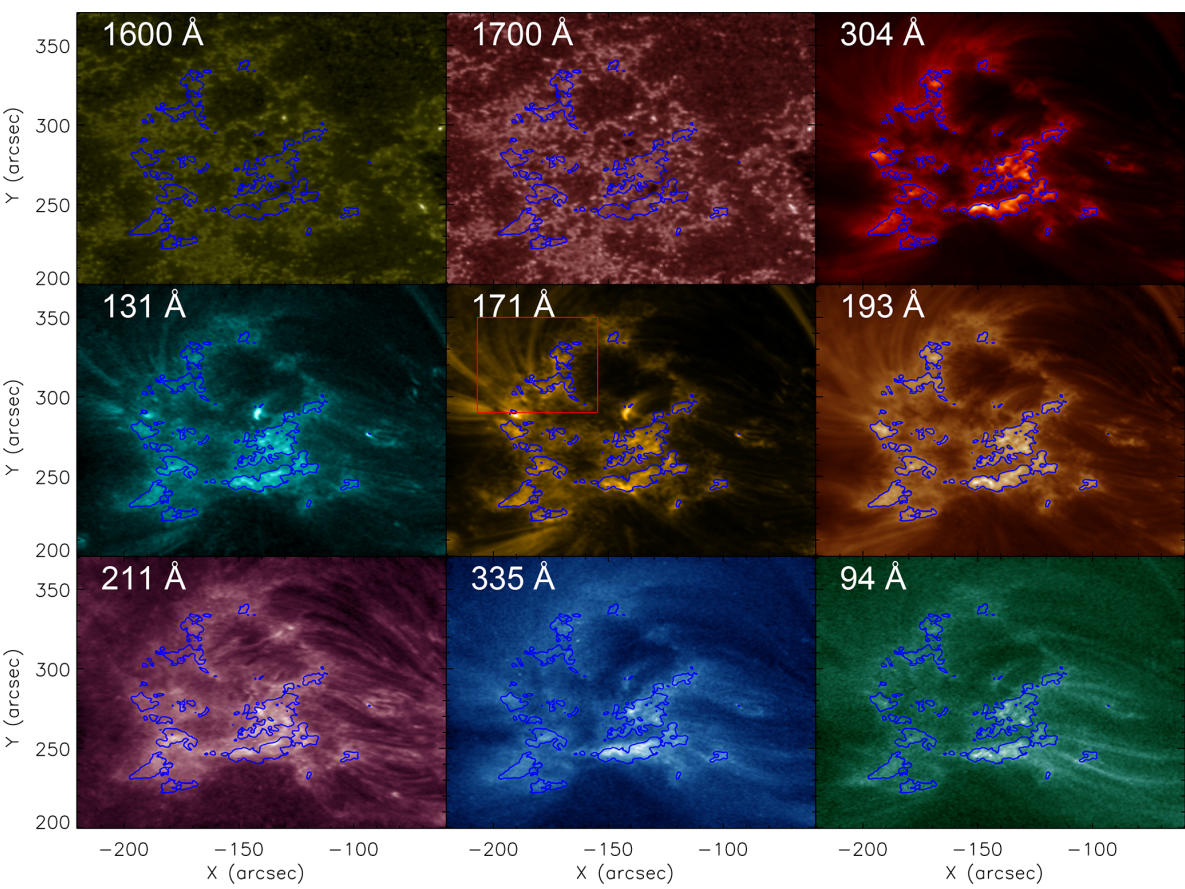

Figure 2. Mosaic representation of the zoomed-in view of the region of interest at different wavelengths of SDO-AIA, as mentioned in the corresponding panels.

(a)
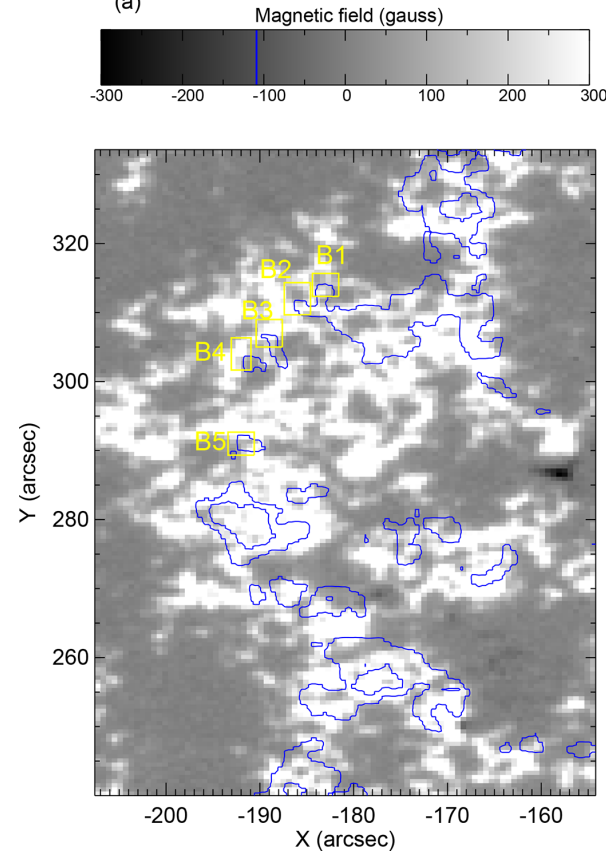

(b)
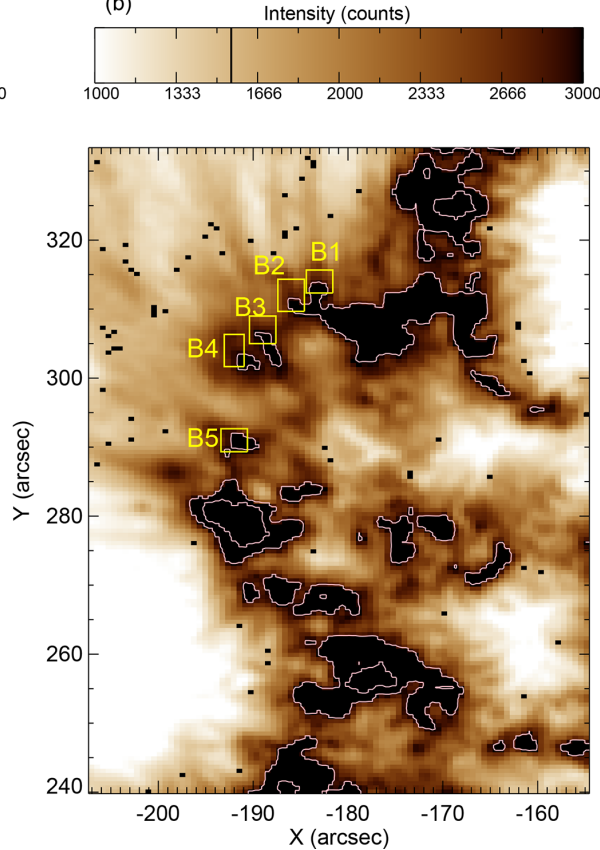

Figure 3. (a) HMI map indicating the magnetic polarities at the moss region, indicted by blue contours. (b) Identification of the foot points of quiescent loops anchored at the moss regions. The different small boxes that are taken at the foot points of the individual loop strands are shown in both the panels. 

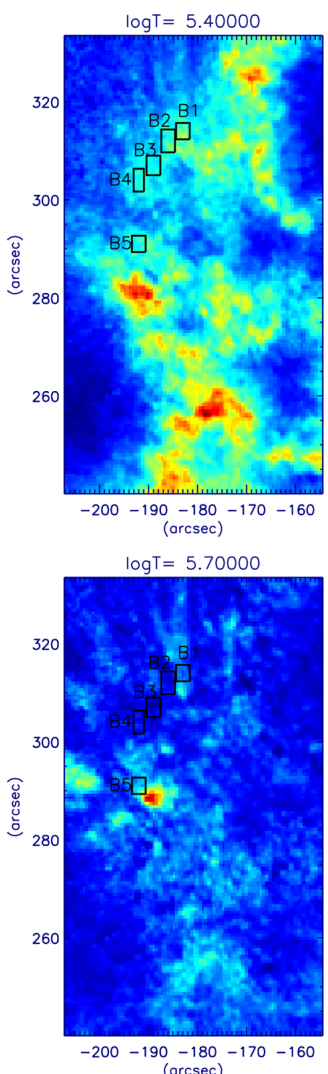
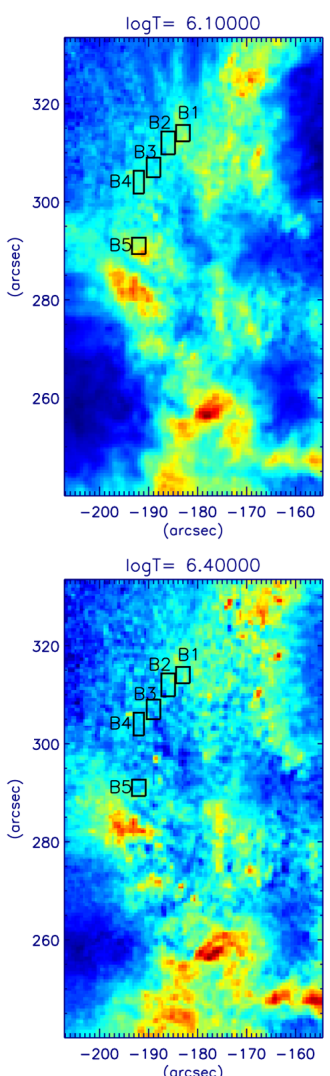
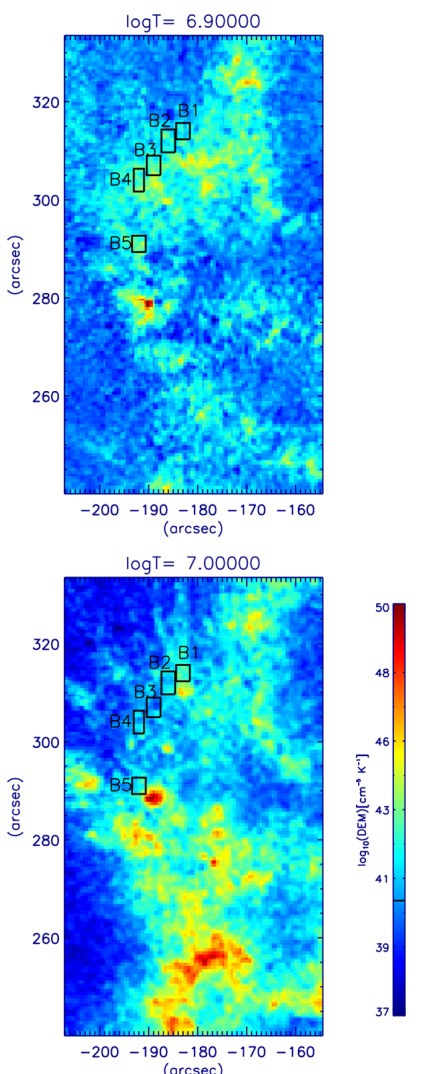

Figure 4. Differential emission measure maps of the plage region containing the moss associated to foot points of quiescent coronal loop systems.

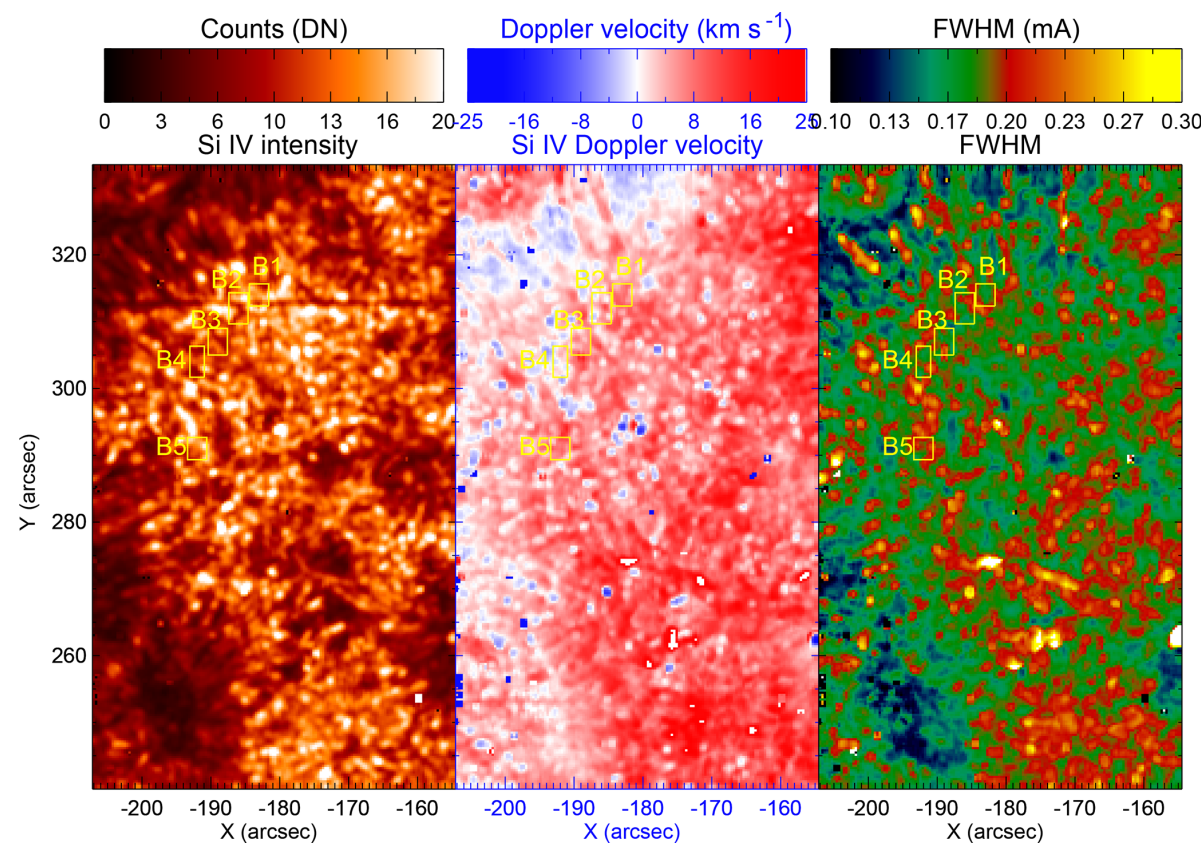

Figure 5. The different parametric plots of Si IV (1393.78 A) line, with the foot points of the quiescent coronal loop systems being indicated by different boxes. DN refers to the data number. 

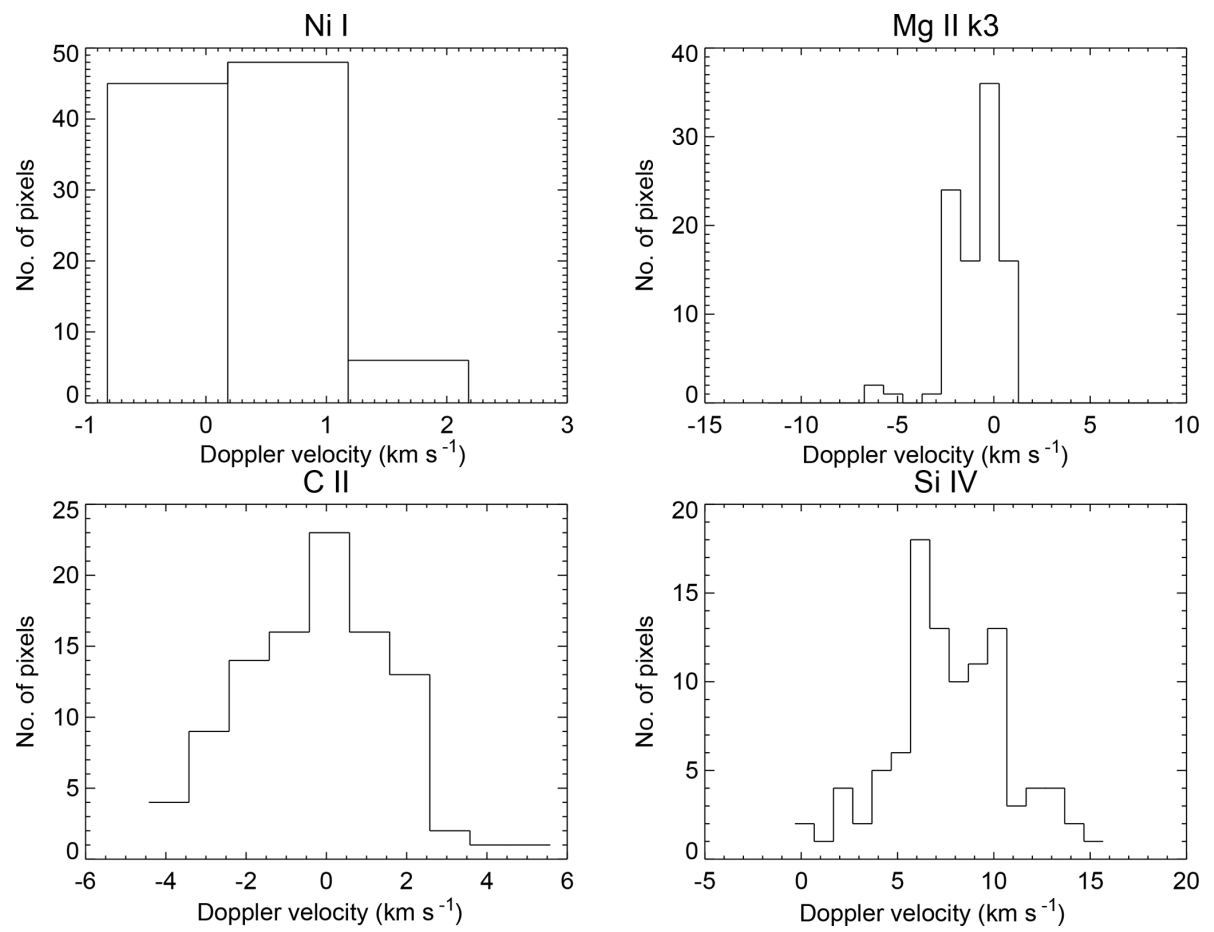

Figure 6. The velocity distributions for different spectral lines corresponding to different temperatures at box B1.
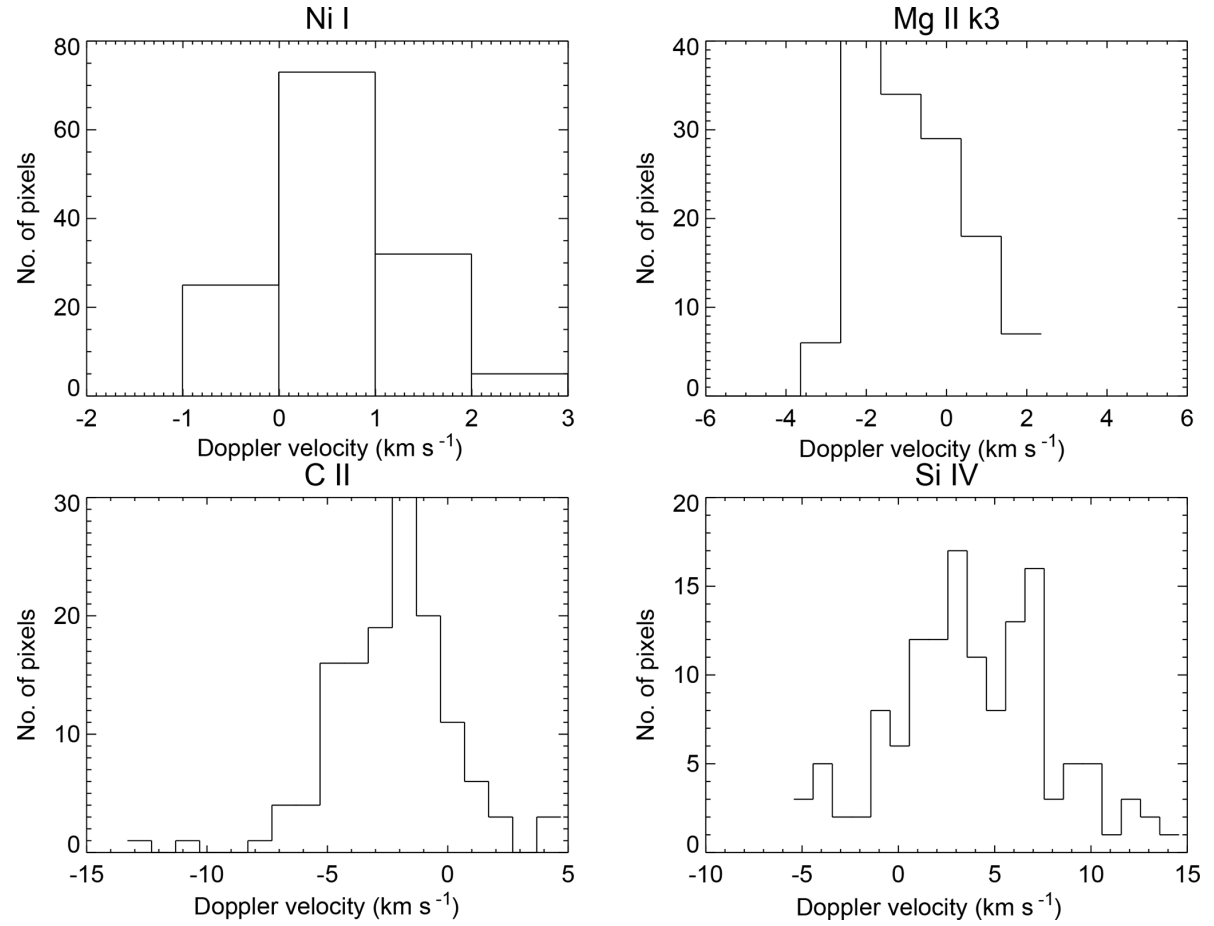

Figure 7. The velocity distributions for different spectral lines corresponding to different temperatures at box B2. 

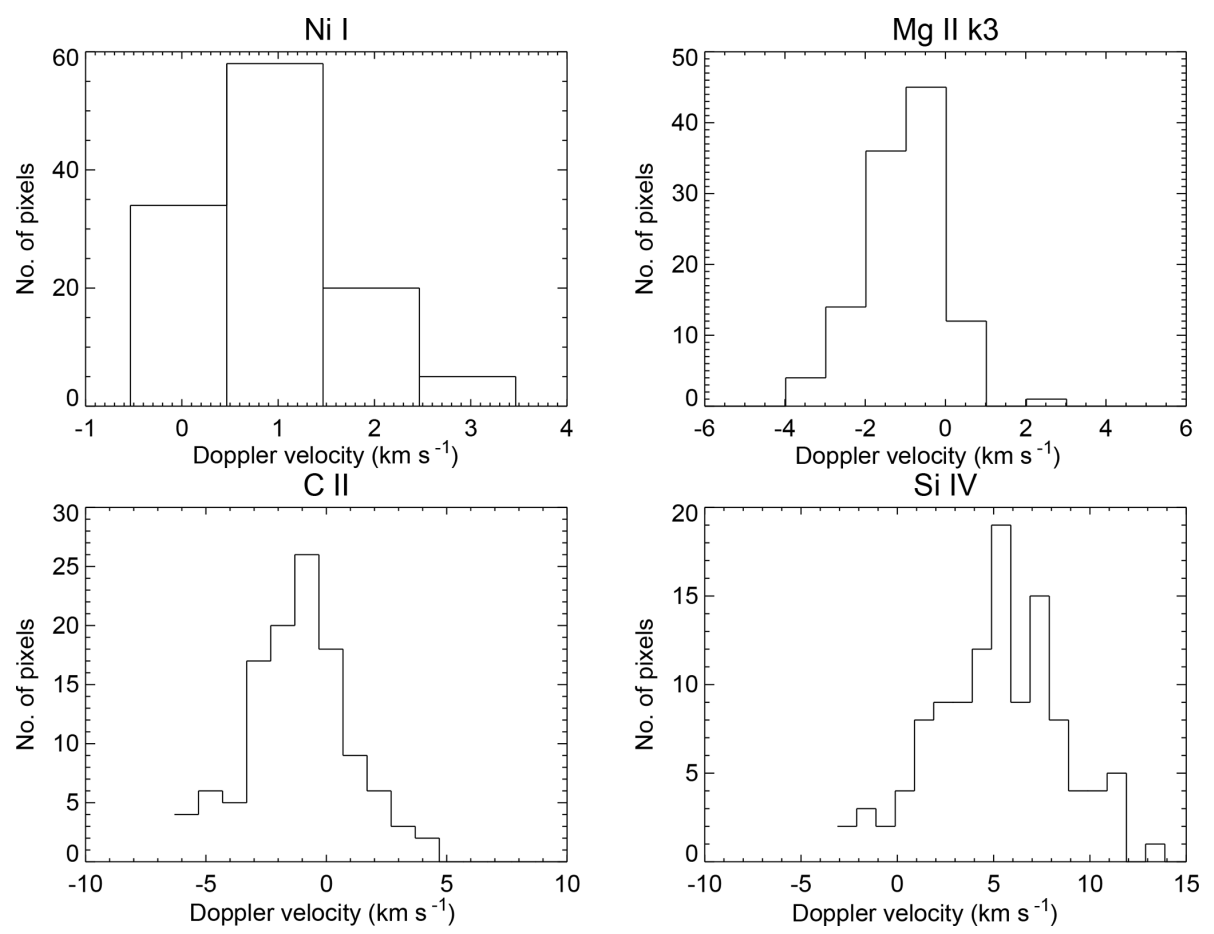

Figure 8. The velocity distributions for different spectral lines corresponding to different temperatures at box B3.

our analysis are not visible, since quiescent loops are dominated by emissions from the temperatures ranging from 0.7 to $1 \mathrm{MK}$, corresponding to SDO-AIA filters.

Figure 3a shows the line-of-sight magnetogram of the region of interest (ROI) taken from the Helioseismic Magnetic Imager (HMI) aboard SDO (HMI/SDO), indicating the magnetic polarities at the moss region as well as foot points of the quiescent coronal loops. Figure $3 \mathrm{~b}$ is the emission of the $193 \AA$ line, plotted in reverse color to identify the foot points. The different boxes of different sizes are then chosen around the foot points to cover the full strand of loop.

Figure 5 shows the parametric plots of the SiIV (1393.7604 ̊̊), showing the intensity, Doppler velocity, and FWHM (full width at half maximum) maps where the values are indicated by the color bars over the plots. The Doppler velocity maps show that the TR is dominated by red shifts even in the plage region surrounding the moss in which foot points were taken for our analysis.

Figure 4 shows the differential emission measure (DEM) maps of the ROI, derived by using the automated method discussed by Hannah and Kontar (2012) in which plasma emission at different temperatures is shown. The color bar indicates the range of the DEM values. These maps show the presence of multi-thermal plasma at the foot points of the quiescent coronal loop systems shown by the boxes around it.

The Doppler velocity distribution is thus explored at different locations, labeled as B1, B2, B3, B4, and B5. Positive values (red shifts) represent downflows, while the negative values (blue shifts) indicate upflows. The Doppler velocity at each pixel in first box (B1) for different spectral lines is then shown in Fig. 6. The velocity distribution for Ni I shows the spread around $0 \mathrm{~km} \mathrm{~s}^{-1}$, ranging from -0.8 to $+2.2 \mathrm{~km} \mathrm{~s}^{-1}$. $\mathrm{Mg}$ II k shows the velocities, ranging from -5 to $+1 \mathrm{~km} \mathrm{~s}^{-1}$, while C II ranges from -5 to $+5 \mathrm{~km} \mathrm{~s}^{-1}$. Si IV shows red shifts with Doppler velocities ranging from 0 to $+15 \mathrm{~km} \mathrm{~s}^{-1}$. The histogram of the Doppler velocity for different spectral lines indicates the red shifts in the Si IV line and very small or negligible flows at Ni I, Mg II k, and C II.

Similarly, such Doppler velocity distribution is shown in Figs. 7, 8, 9, and 10 for different boxes, labeled as B2, B3, $\mathrm{B} 4$, and B5.

Figure 11 shows the average Doppler shift of different spectral lines as a function of their temperatures for different boxes chosen at the foot points of the loops. Ni I (2799.47 $\mathrm{\AA})$ corresponds to the upper photosphere, and $\mathrm{Mg}$ II k yields emission ranging from the mid-chromosphere to upper chromosphere. The core defined by $(\mathrm{k} 3)$ forms little higher than the wings at $200 \mathrm{~km}$ below TR (Leenarts et al., 2013). The C II core yields emission from $2.1 \mathrm{Mm}$, while Si IV corresponds to the TR emission (Rathore et al., 2015).

The Doppler velocity of the Ni I line has negligible values, indicating almost no flows $\left(0.27\right.$ to $\left.0.70 \mathrm{~km} \mathrm{~s}^{-1}\right)$, corresponding to the photospheric region. The blue shifts (upflows) show small increments for B2, B4, and B4 ( -0.11 to $-0.31 \mathrm{~km} \mathrm{~s}^{-1}$ ), while they remain almost the same for B1 $\left(0.16 \mathrm{~km} \mathrm{~s}^{-1}\right)$ and B5 $\left(0.80 \mathrm{~km} \mathrm{~s}^{-1}\right)$ up to the formation temperature of $\mathrm{Mg}$ II k. The C II line shows considerable blue 

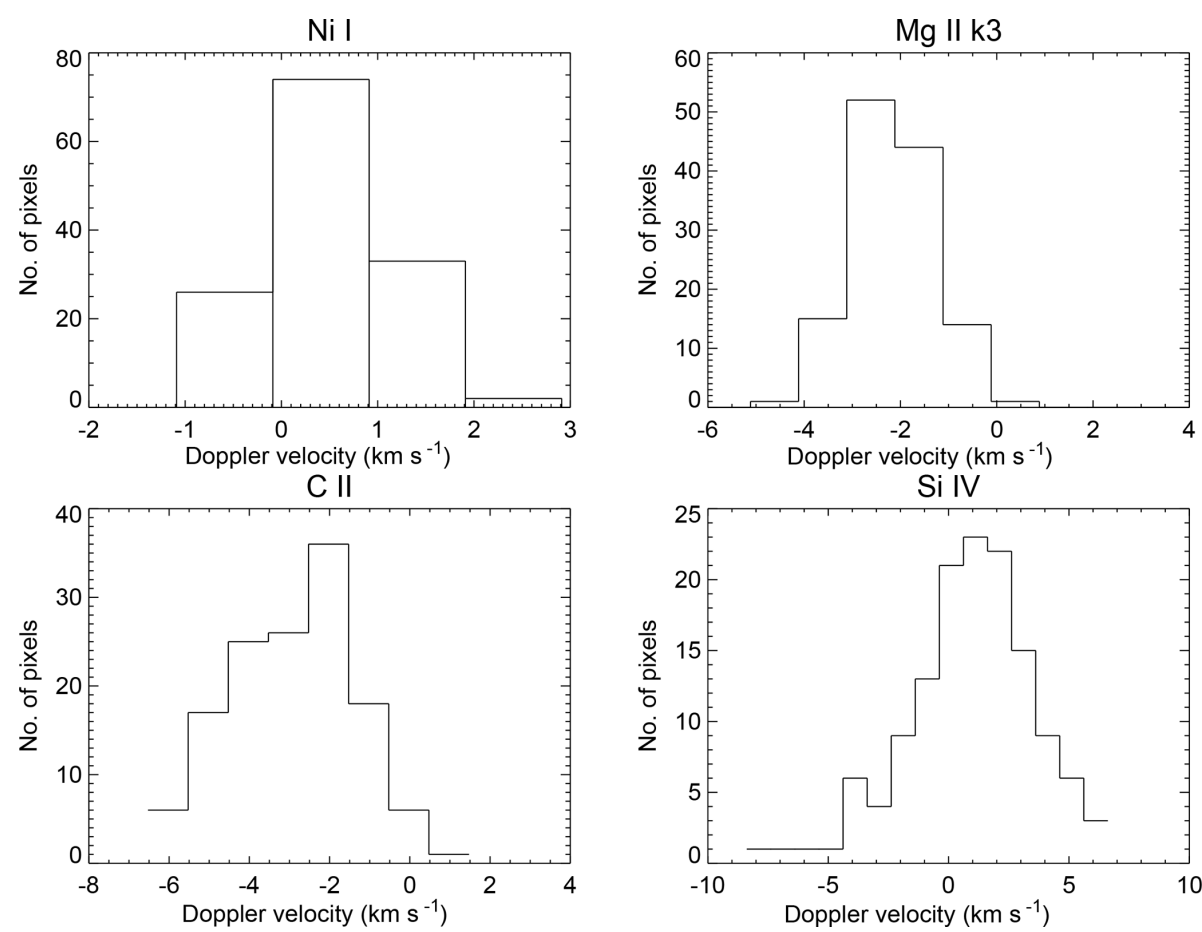

Figure 9. The velocity distributions for different spectral lines corresponding to different temperatures at box B4.
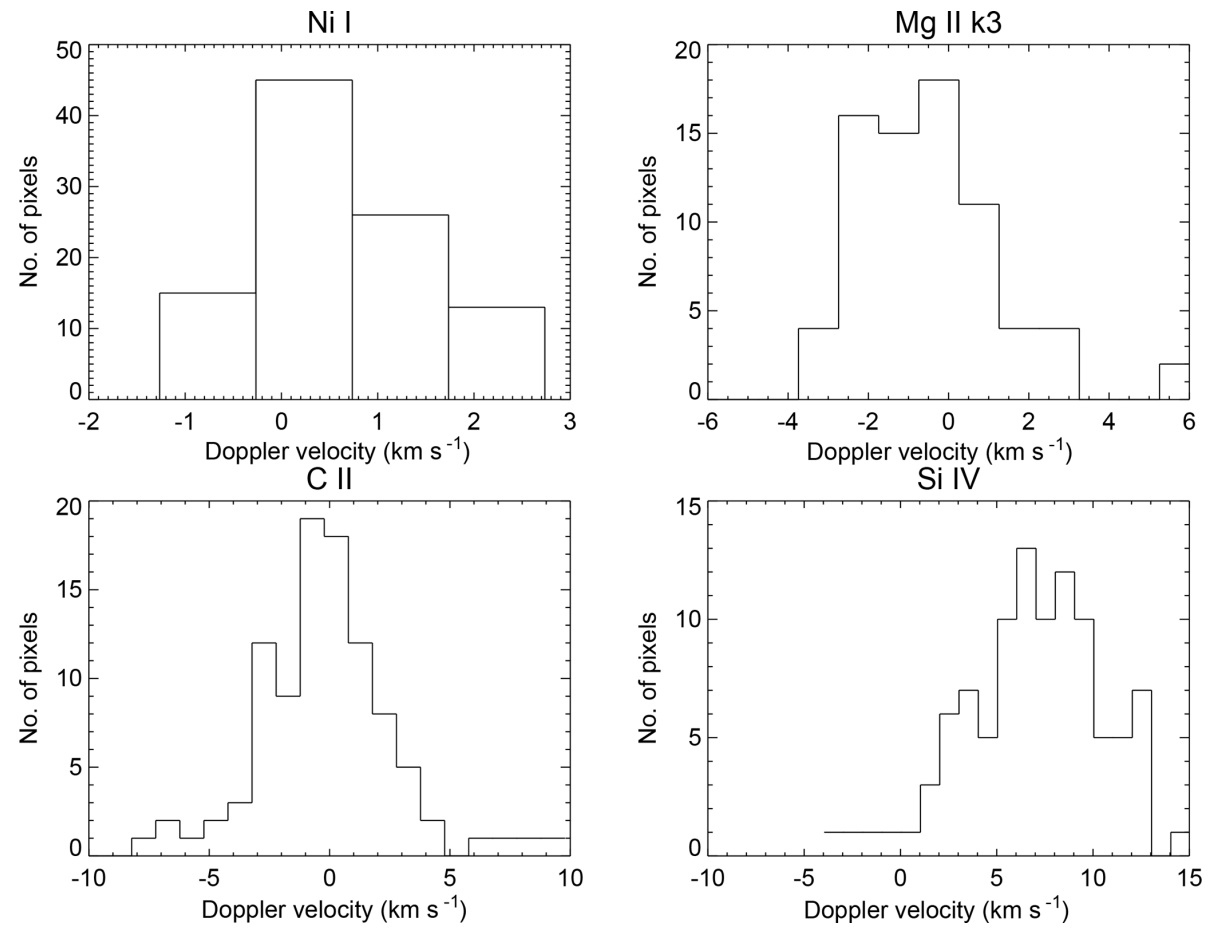

Figure 10. The velocity distributions for different spectral lines corresponding to different temperatures at box B5. 


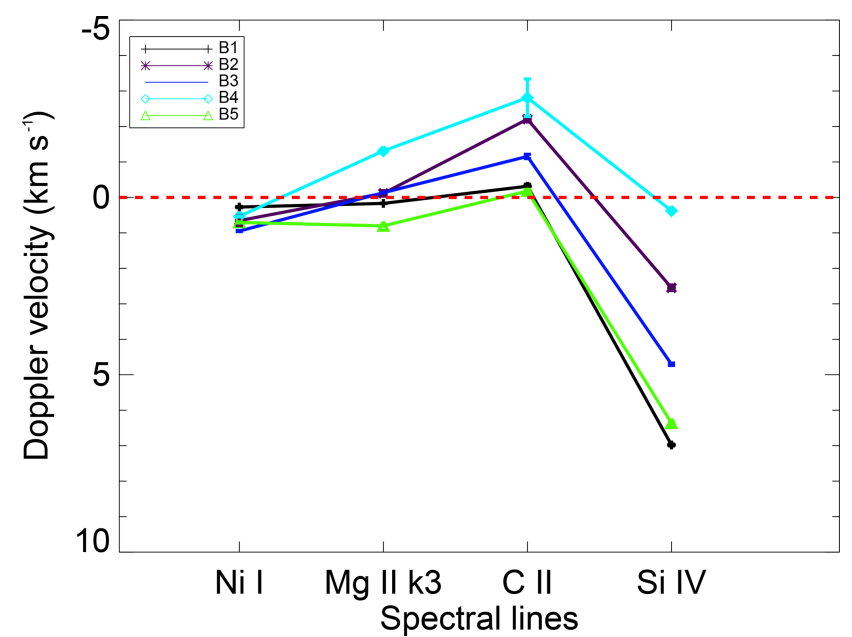

Figure 11. Average Doppler velocity variations for different spectral lines dominating at different heights in the solar atmosphere for boxes B1, B2, B3, B4, and B5 at the foot points of quiescent coronal loops.

shifts (upflows) of -0.17 to $-2.81 \mathrm{~km} \mathrm{~s}^{-1}$, but it is still negligible when compared to chromospheric flows. The Doppler velocity variation at $\mathrm{Si}$ IV shows prevalent red shifts (downflows) at all the locations corresponding to TR flows ( 0.37 to $6.97 \mathrm{~km} \mathrm{~s}^{-1}$ ). The $1 \sigma$ error is shown as error bars, which is difficult to visualize in Fig. 11, owing to its very small values.

\section{Discussions and conclusions}

The co-spatial multi-spectral Doppler velocity trend at the foot points of quiescent coronal loops was studied. The Doppler velocity variation shows small flows (upflows and downflows) for $\mathrm{Ni} \mathrm{I}$ and $\mathrm{Mg}$ II $\mathrm{k}$ in the photospheric as well as the chromospheric region. C II shows very blue shifts $(-0.1$ to $-2.81 \mathrm{~km} \mathrm{~s}^{-1}$ ), indicating small upflows at the upper chromospheric region. The Doppler velocities then change to red shifts at the formation temperature of the Si IV line, corresponding to the TR.

It was previously shown that the moss regions show significant red shifts (downflows) in the TR, explaining the lowfrequency heating (Bradshaw and Cargill, 2010). The highand low-frequency mechanisms depend on the time taken by the loops to cool down when compared to heating frequency (Tripathi et al., 2008).

Our study of the flows at the quiescent coronal loops shows similar characteristics to the dynamically active loops, though the velocity values are smaller. The plasma predominantly shows red shifts at TR temperatures which corroborate with the low-frequency heating of loops in the coronal part of the solar atmosphere. These observations thus agree with the coronal loops heated up by low-frequency nanoflares via an impulsive heating mechanism. Also, Patsourakos and Klimchuk (2006) observed the symmetric profiles for steady heat- ing in the loops. Though it is possible to have asymmetries in the individual profile for which velocity distribution has been observed, our speculation supports the nanoflare-driven impulsive heating mechanism for the quiescent coronal loops.

The asymmetries may also cause the Doppler variation in the spectral profiles due to a difference in the pressures. (Mariska and Boris, 1983). Thus, other possibilities cannot be ruled out.

Data availability. All the data which we have used in the paper can be accessed publicly at http://www.iris.lmsal.com/search/ (last access: 19 July 2019).

Author contributions. All authors have contributed in an equal manner.

Competing interests. The authors declare that they have no conflict of interest.

Special issue statement. This article is part of the special issue "Solar magnetism from interior to corona and beyond". It is a result of the Dynamic Sun II: Solar Magnetism from Interior to Corona, Siem Reap, Angkor Wat, Cambodia, 12-16 February 2018.

Acknowledgements. We acknowledge the use of IRIS observations. IRIS is a NASA Small Explorer mission developed and operated by the Lockheed Martin Solar and Astrophysics Laboratory (LMSAL), with mission operations executed at the NASA Ames Research Center and major contributions to downlink communications funded by the Norwegian Space Centre (NSC; Norway) through an ESA PRODEX contract.

Financial support. Yamini K. Rao is fully supported by the financial grant from the ISRO RESPOND project.

Review statement. This paper was edited by Viktor Fedun and reviewed by two anonymous referees.

\section{References}

Bradshaw, S. J. and Cargill, P. J.: A New Enthalpy-Based Approach to the Transition Region in an Impulsively Heated Corona, Astrophys. J. Lett., 710, L39, https://doi.org/10.1088/20418205/710/1/L39, 2010.

Brooks, D. H., Warren, H. P., and Young, P. R.: EUV Spectral Line Formation and the Temperature Structure of Active Region Fan Loops: Observations with Hinode/EIS and SDO/AIA, Astrophys. J., 730, 85, https://doi.org/10.1088/0004-637X/730/2/85, 2011. 
Del Zanna, G.: Flows in active region loops observed by Hinode EIS, Astron. Astrophys., 481, L49, https://doi.org/10.1051/00046361:20079087, 2008.

Del Zanna, G. and Mason, H. E.: Solar active regions: SOHO/CDS and TRACE observations of quiescent coronal loops Astron. Astrophys., 406, 1089, https://doi.org/10.1051/00046361:20030791, 2003.

Del Zanna, G., O’Dwyer, B., and Mason, H. E.: SDO AIA and Hinode EIS observations of "warm" loops, Astron. Astrophys., 535, A46, https://doi.org/10.1051/0004-6361/201117470, 2011.

De Moortel, I. and Browning, P.: Recent advances in coronal heating, Philos. T. R. Soc. Lond. S-A, 373, 20140269, https://doi.org/10.1098/rsta.2014.0269, 2015.

De Pontieu, B., Title, A. M., Lemen, J. R., Kushner, G. D., Akin, D. J., Allard, B., Berger, T., Boerner, P., Cheung, M., Chou, C., Drake, J. F., Duncan, D. W., Freeland, S., Heyman, G. F., Hoffman, C., Hurlburt, N. E., Lindgren, R. W., Mathur, D., Rehse, R., Sabolish, D., Seguin, R., Schrijver, C. J., Tarbell, T. D., Wülser, J.-P., Wolfson, C. J., Yanari, C., Mudge, J., Nguyen-Phuc, N., Timmons, R., van Bezooijen, R., Weingrod, I., Brookner, R., Butcher, G., Dougherty, B., Eder, J., Knagenhjelm, V., Larsen, S., Mansir, D., Phan, L., Boyle, P., Cheimets, P. N., DeLuca, E. E., Golub, L., Gates, R., Hertz, E., McKillop, S., Park, S., Perry, T., Podgorski, W. A., Reeves, K., Saar, S., Testa, P., Tian, H., Weber, M., Dunn, C., Eccles, S., Jaeggli, S. A., Kankelborg, C. C., Mashburn, K., Pust, N., Springer, L., Carvalho, R., Kleint, L., Marmie, J., Mazmanian, E., Pereira, T. M. D., Sawyer, S., Strong, J., Worden, S. P., Carlsson, M., Hansteen, V. H., Leenaarts, J., Wiesmann, M., Aloise, J., Chu, K.-C., Bush, R. I., Scherrer, P. H., Brekke, P., Martinez-Sykora, J., Lites, B. W., McIntosh, S. W., Uitenbroek, H., Okamoto, T. J., Gummin, M. A., Auker, G., Jerram, P., Pool, P., and Waltham, N.: The Interface Region Imaging Spectrograph (IRIS), Solar Phys., 289, 2733, https://doi.org/10.1007/s11207-014-0485-y, 2014.

Fletcher, L. and De Pontieu, B.: Plasma Diagnostics of Transition Region "Moss" using SOHO/CDS and TRACE, Astrophys. J. Lett., 520, L135, https://doi.org/10.1086/312157, 1999.

Hannah, I. G. and Kontar, E. P.: Differential emission measures from the regularized inversion of Hinode and SDO data, Astron. Astrophys., 539, A146, https://doi.org/10.1051/00046361/201117576, 2012.

Huang, Z., Xia, L., Li, B., and Madjarska, M. S.: Cool Transition Region Loops Observed by the Interface Region Imaging Spectrograph, Astrophys. J., 810, 46, https://doi.org/10.1088/0004637X/810/1/46, 2015.
Klimchuk, J. A.: On Solving the Coronal Heating Problem, Solar Phys., 234, 41, https://doi.org/10.1007/s11207-006-0055-z, 2006.

Leenaarts, J., Pereira, T. M. D., Carlsson, M., Uitenbroek, H., and De Pontieu, B.: The Formation of IRIS Diagnostics. II. The Formation of the Mg II h\&k Lines in the Solar Atmosphere, Astrophys. J., 772, 90, https://doi.org/10.1088/0004-637X/772/2/90, 2013.

Lemen, J. R., Title, A. M., and Akin, D. J.: The Atmospheric Imaging Assembly (AIA) on the Solar Dynamics Observatory (SDO), Solar Phys., 275, 17, https://doi.org/10.1007/s11207-011-97768, 2012.

Mariska, J. T. and Boris, J. P.: Dynamics and spectroscopy of asymmetrically heated coronal loops, Astrophys. J., 267, 409, https://doi.org/10.1086/160879, 1983.

Patsourakos, S. and Klimchuk, J. A.: Nonthermal Spectral Line Broadening and the Nanoflare Model, Astrophys. J., 647, 1452, https://doi.org/10.1086/505517, 2006.

Rao, Y. K., Srivastava, A. K., Kayshap, P., Wilhelm, K., and Dwivedi, B. N.: Plasma Flows in the Cool Loop Systems, Astrophys. J., 874, 56, https://doi.org/10.3847/1538-4357/ab06f5, 2019.

Rathore, B., Carlsson, M., Leenaarts, J., and De Pontieu, B.: The Formation of IRIS Diagnostics. VI. The Diagnostic Potential of the C II Lines at $133.5 \mathrm{~nm}$ in the Solar Atmosphere, Astrophys J., 811, 81, https://doi.org/10.1088/0004-637X/811/2/81, 2015.

Reale, F.: Coronal Loops: Observations and Modeling of Confined Plasma, Living Rev. Sol. Phys., 11, 4, https://doi.org/10.12942/lrsp-2014-4, 2014.

Tripathi, D., Mason, H. E., Young, P. R., and Del Zanna, G.: Density structure of an active region and associated moss using Hinode/EIS, Astron. Astrophys., 481, L53, https://doi.org/10.1051/0004-6361:20079034, 2008.

Warren, H. P. and Brooks, D. H.: The Temperature and Density Structure of the Solar Corona. I. Observations of the Quiet Sun with the EUV Imaging Spectrometer on Hinode, Astrophys. J., 700, 762, https://doi.org/10.1088/0004-637X/700/1/762, 2009.

Young, P. R., O’Dwyer, B., and Mason, H. E.: Velocity Measurements for a Solar Active Region Fan Loop from Hinode/EIS Observations, Astrophys. J., 744, 14, https://doi.org/10.1088/0004637X/744/1/14, 2012. 